\title{
Modeling the Particle Transport and Ion Production in a RF Driven Negative Hydrogen Ion Source for ITER NBI
}

\author{
D. Wünderlich, P. McNeely, L. Schiesko, U. Fantz, P. Franzen and the \\ NNBI-Team
}

\author{
Max-Planck-Institut für Plasmaphysik (IPP), EURATOM Association, \\ Boltzmannstr. 2, D-85748 Garching, Germany
}

\begin{abstract}
NBI systems based on the generation, acceleration and neutralization of negative hydrogen or deuterium ions are foreseen for heating and current drive in ITER. Since 2007 the $\mathrm{RF}$ driven ion source prototype developed at the Max Planck Institut für Plasmaphysik (IPP) is the ITER reference source.

Negative hydrogen ions are predominantly produced by conversion of hydrogen atoms or positive ions at the cesiated surface of the plasma grid (PG), the first grid of a three-grid extraction system. In interaction of the charged plasma particles with a magnetic filter field and a bias voltage applied to the PG an extended boundary layer evolves - a several centimeters thick plasma volume close to the PG relevant for the transport and extraction of the negative hydrogen ions.

Aim of this work is to investigate the relative relevance of the negative hydrogen ion production by conversion of atoms compared to conversion of positive ions. The ratio of these two conversion channels is connected to the homogeneity of the extracted negative ion beam. Three codes dealing with the relevant physical processes in the boundary layer are introduced and applied. The most recent code results indicate that conversion of atoms is the dominant production channel and that for ensuring a homogeneous negative ion beam production the gas uniformity close to the PG is of greater importance than the plasma uniformity.
\end{abstract}

Keywords: Negative hydrogen ion source, beam homogeneity, Monte Carlo code, PIC code. PACS: 29.25.Ni, 52.27.Cm, 52.65.Cc, 52.65.Pp, 52.65.Rr.

\section{INTRODUCTION}

For heating and current drive in ITER two NBI beamlines are planned with a heating power of $16.5 \mathrm{MW}$ each [1,2]. Since 2007 the RF source for negative hydrogen ions developed at IPP Garching [3,4] is the ITER reference source [1].

Figure 1 shows a schematic view of the IPP prototype ion source. The plasma is generated inductively ( $f_{\mathrm{RF}}=1 \mathrm{MHz}$, typical RF power: 70 to $100 \mathrm{~kW}$ ) in the driver and then expands into the expansion region. In order to reduce the destruction rate of negative ions and the amount of co-extracted electrons the electrons are cooled down (to $\mathrm{T}_{\mathrm{e}} \approx 1 \mathrm{eV}$ at electron densities around $10^{17} \mathrm{~m}^{-3}$ ) close to the extraction system by means of a magnetic filter field. The extraction and acceleration system consists of three grids: the plasma grid (PG), the extraction grid and the grounded grid. In order to reduce the ratio of co-extracted electrons to extracted negative ions the PG is 
positively biased with respect to the source body [3]. The co-extracted electrons are removed from the extracted beam by means of electron deflection magnets embedded into the extraction grid.

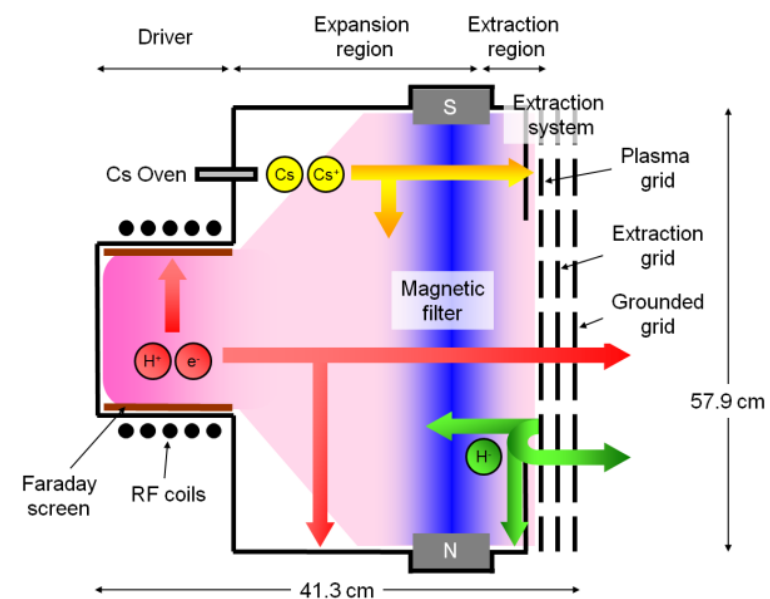

FIGURE 1. Schematic view of the IPP prototype negative hydrogen ion source.

The production of negative hydrogen or deuterium ions occurs mainly at the surface of the PG, which is covered with a thin cesium layer in order to reduce the surface work function $[3,5,6]$. Two channels are possible for this production process: transfer of electrons from the surface to approaching hydrogen atoms [7] or positive hydrogen ions [8]. The latter are neutralized in a first step by an Auger process close to the surface; molecular ions are dissociated first.

The gas distribution during the plasma pulses is influenced by neutral depletion [9]. Although no spatially resolved measurements exist, some effect of neutral depletion on the uniformity of the atomic hydrogen density close to the PG can be expected. On the other hand, the plasma and thus also the positive hydrogen ion flux in front of the extraction system can be non-uniform [10]. The reason is a vertical plasma drift, caused by the magnetic filter field. The degree of this non-uniformity can be influenced over a wide range by the bias voltage [11]. Since the described nonuniformities of gas and plasma are caused by different physical effects, the spatial distribution of the atomic hydrogen density over the PG surface is not necessarily identical to the one of the plasma density.

For ITER NBI an inhomogeneity of the accelerated negative ion beam smaller than $10 \%$ is required. Depending on the relative relevance of the two surface production channels the beam homogeneity is either determined by the neutral gas uniformity or the plasma uniformity or a combination of both. An additional role can be played by inhomogeneities of the conversion yield over the PG surface. It has been shown by beam emission spectroscopy that the extracted beam is much more homogeneous than the plasma [12]. But up to now, no detailed theoretical investigations on this topic have been performed.

An important role is played by the boundary layer [13]: in this several centimeters thick layer close to the PG surface the relevant physics for the production, transport and extraction of the negative hydrogen ions takes place. Figure 2 shows a schematic representation of the boundary layer and the relevant physical processes. 
In the last years several codes have been developed at IPP which investigate these processes separately. This paper presents calculations which interconnect three of these codes in order to analyze the interplay of the different physical processes:

Firstly, the Monte Carlo transport code Protonflow3d calculates the proton energy distribution function (EDF) in the boundary layer. Secondly, the PIC code Bacon calculates the transport of hydrogen atoms and positive ions through the plasma sheath towards the PG surface, the surface generation process and the transport of the surface generated negative ions towards the sheath edge. Bacon uses as input a measured atomic EDF and the proton EDF determined by ProtonFlow3d. Thirdly, the Monte Carlo transport code TrajAn is applied to investigate the extraction probability of the negative hydrogen ions on their way from the sheath edge towards the extraction apertures.

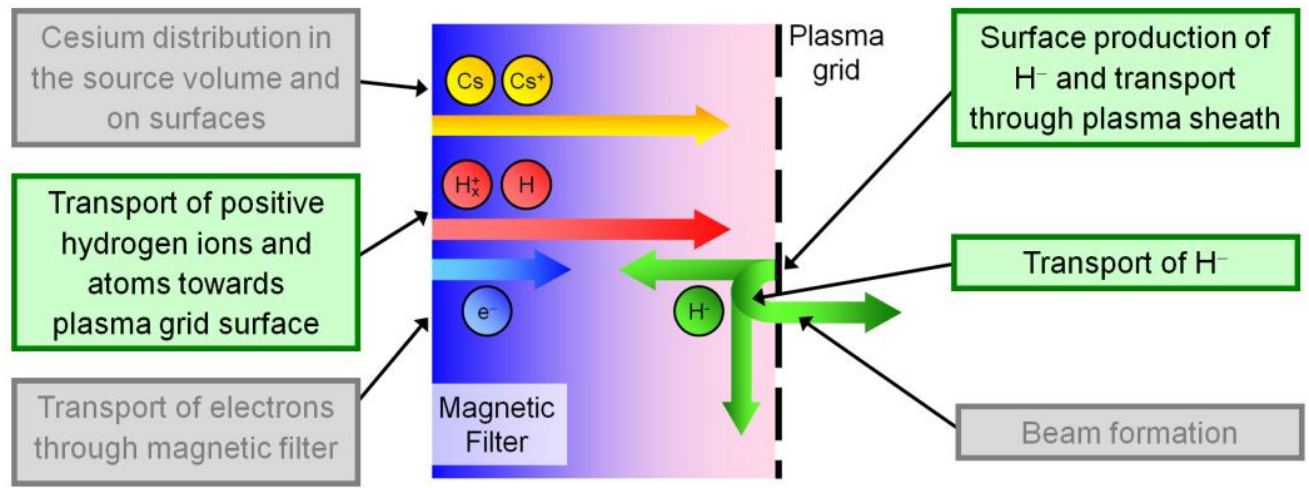

FIGURE 2. Schematic representation of the boundary layer close to the PG surface. Indicated by boxes are the relevant physical processes. Shown in green are the processes dealt with within this paper.

The combination of the three codes allows to determine the relative contribution of the two surface conversion channels to the extracted negative ions. Thus, a statement will be possible if the neutral gas uniformity or the plasma uniformity is a key parameter for the homogeneity of the negative ion beam.

\section{DESCRIPTION OF THE USED CODES}

\section{The Monte Carlo Transport Code ProtonFlow3d}

The three dimensional transport code ProtonFlow3d was developed in order to investigate the transport of charged particles from their point of birth in the driver volume towards the PG [14]. ProtonFlow3d is a test particle Monte Carlo code. Implemented is the geometry of the IPP prototype ion source as well as - as far as possible - the topology of the electrostatic field.

In the code, test protons are generated in the driver. Then the code follows the trajectory until the particle is destroyed by a collision with a background particle or hits one of the ion source side walls or the PG surface. The current version of the code does not account for $\mathrm{H}_{2}{ }^{+}$and $\mathrm{H}_{3}{ }^{+}$. Although due to different collision cross sections and different particle masses the calculation results for these heavier ions will differ 
from the results for protons, the basic behavior of the three different positive ion species should be comparable.

The motion of the test particles is divided into small time steps. For each time step the electrostatic field strength is calculated from the profile of the electrostatic potential. The field strength is then substituted into the Lorentz equation in order to calculate the particle velocity after the time step. The electrostatic potential is defined externally. Since experimental data on the $3 \mathrm{~d}$ field structure inside the ion source is scarce, only the measured axial dependence of the electric field is taken into account by ProtonFlow $3 \mathrm{~d}$. A full $3 \mathrm{~d}$ potential map would include the plasma sheath at the ion source side walls as well as potential gradients perpendicular to the axial direction, resulting in an additional deflection of the proton trajectories towards the side walls. The axial potential profiles used are generated by scaling and merging the results for the driver field from [15] and for the expansion region from [10]. Figure 3 shows the resulting axial profile for high power operation $\left(\mathrm{P}_{\mathrm{HF}}=70 \mathrm{~kW}, \mathrm{p}_{\mathrm{fill}}=0.45 \mathrm{~Pa}\right)$.

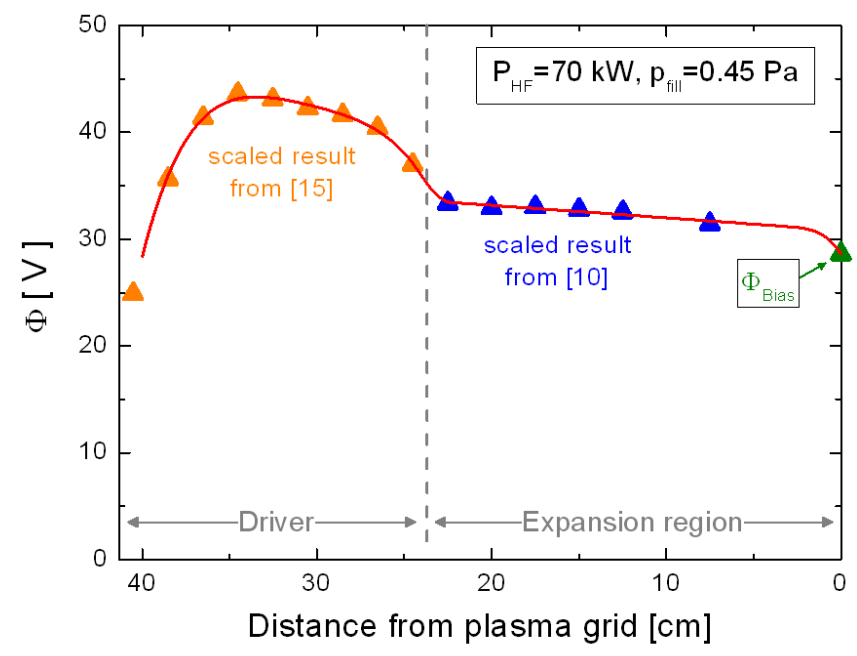

FIGURE 3. Axial potential profile for high power operation of the IPP prototype ion source.

A Monte Carlo module is invoked for each time step in order to decide whether the particle participates in an elastic or inelastic collision. Included in Protonflow $3 \mathrm{~d}$ are the six most relevant collision processes of protons with the background gas, i.e. the six collisions with the largest collision frequency. These reactions are listed in Table 1.

TABLE 1. Reaction processes implemented into Protonfl ow 3 d.

\begin{tabular}{lccc}
\hline Reaction & Process & Ref. & Comment \\
\hline Momentum transfer with $\mathrm{H}$ (elastic) & $\mathrm{H}^{+}+\mathrm{H} \rightarrow \mathrm{H}^{+}+\mathrm{H}$ & {$[16]$} & \\
Momentum transfer with $\mathrm{H}_{2}$ (elastic) & $\mathrm{H}^{+}+\mathrm{H}_{2} \rightarrow \mathrm{H}^{+}+\mathrm{H}_{2}$ & {$[17]$} & \\
Charge exchange with $\mathrm{H}$ & $\mathrm{H}^{+}+\mathrm{H} \rightarrow \mathrm{H}+\mathrm{H}^{+}$ & {$[18]$} & \\
Charge exchange with $\mathrm{H}_{2}$ & $\mathrm{H}^{+}+\mathrm{H}_{2} \rightarrow \mathrm{H}+\mathrm{H}_{2}^{+}$ & {$[18]$} & $\mathrm{T}_{\text {vib }}=5000 \mathrm{~K}$ \\
Vibrational excitation & $\mathrm{H}^{+}+\mathrm{H}_{2}(\mathrm{v}=0) \rightarrow \mathrm{H}^{+}+\mathrm{H}_{2}(\mathrm{v}=1 \ldots 3)$ & {$[17]$} & \\
Rotational excitation & $\mathrm{H}^{+}+\mathrm{H}_{2}(\mathrm{~J}=0) \rightarrow \mathrm{H}^{+}+\mathrm{H}_{2}(\mathrm{~J}=2)$ & {$[17]$} & \\
\hline
\end{tabular}

The reaction probability for charge exchange with hydrogen molecules strongly depends on the vibrational excitation of the molecule. In order to obtain an effective 
cross section, the cross sections for the different vibrational quantum numbers have been averaged, using a relative vibrational population characterized by a vibrational temperature of $\mathrm{T}_{\mathrm{vib}}=5000 \mathrm{~K}$. The choice of this vibrational temperature is justified by optical emission spectroscopy measurements in the driver and in the expansion region [19].

For most collision reactions no differential cross section is available that would depict the collision probability as a function of the relative particle energy and the scattering angle. The existing differential cross sections for vibrational [20] and rotational [21] excitation are available only for a too small number of different collision energies. Thus, for all reactions the angular distribution suggested in [22] for elastic ion-neutral collisions is being applied.

Measured values from [9] have been used as densities of the background particles involved in the collisions $\left(\mathrm{n}\left(\mathrm{H}_{2}\right)=4.5 \cdot 10^{19} \mathrm{~m}^{-3}, \mathrm{n}(\mathrm{H})=4.5 \cdot 10^{18} \mathrm{~m}^{-3}\right)$.

ProtonFlow $3 \mathrm{~d}$ neglects the influence of the magnetic filter on the motion of the test particles as well as Coulomb collisions. Both effects will lead to a significant deceleration of the protons. It has been shown experimentally that the presence of the filter field results in a reduction of the axial proton velocity by more than a magnitude [23]. This means that the energies calculated by the code for the protons close to the PG represent an upper limit.

The output routines of Protonflow3d deliver the EDF of all protons approaching the PG. An arbitrary number of diagnostic layers, acting as EDF detectors, can be defined in the plasma. In combination, phase space diagrams can be constructed which describe the spatial dependence of the proton EDF between driver and PG surface.

\section{The PIC Code Bacon}

The PIC code Bacon, developed at IPP, calculates the negative ion current densities emitted into the plasma volume at the sheath edge [24]. The code distinguishes between negative ions generated by conversion of atoms and positive ions, respectively. Used as input are the electron density and electron temperature as well as the atomic and the positive ion flux towards the sheath edge.

PIC codes calculate the movement of charged particles in plasmas and the resulting potential distribution in a self-consistent way [25]. This is done by tracking the trajectories of a large number of macro particles (each of which represents a certain number of real particles). Time is divided into small time steps and space is divided into grid cells. For each time step the charge of the particles is projected onto the grid and the electrostatic potential is calculated by solving Poisson's equation.

Bacon is a $1 \mathrm{~d} 3 \mathrm{v}$ code. This means that only one spatial dimension is taken into account while all three velocity components are accounted for. The calculation domain represents a small volume close to the PG. Electrons, protons and positive cesium ions are introduced into the domain at a particle source, depicting the sheath edge, i.e. the transition to the bulk plasma. Like ProtonFlow3d, the current version of Bacon does not account for $\mathrm{H}_{2}{ }^{+}$and $\mathrm{H}_{3}{ }^{+}$. Again, the basic results for the three positive hydrogen ion species should be comparable. 
When protons hit the PG surface, a Monte Carlo module is invoked which determines whether a negative ion is produced or not. The conversion of hydrogen atoms to negative ions is implemented as an additional and constant influx term.

In principle, Bacon can include the effect of magnetic fields on the particle transport. In order to ensure compatibility with the results from ProtonFlow $3 \mathrm{~d}$ no magnetic field was used in the present calculations.

The output routines of Bacon deliver profiles of the electrostatic potential and the charged particle species densities for arbitrary time steps. Additionally given are the temporal evolution of several parameters like the number of macro particles for all particle species, the electrostatic potential at several positions and the particle fluxes measured at user-defined diagnostic layers inside the domain. The negative hydrogen fluxes originating from both surface conversion channels are detected separately at a diagnostic layer at the sheath edge.

\section{The Monte Carlo Transport Code TrajAn}

Prior to extraction, the negative ions emitted at the sheath edge have to be bent back towards the extraction apertures. Two processes are mainly responsible for this change of direction: collisions with the background plasma and interaction with the magnetic fields.

The Monte Carlo transport code TrajAn [26] calculates the trajectories of surface produced negative hydrogen ions from the sheath edge until the ion is either extracted or destroyed by a collision. Included into the code is the geometry of the LAG grid system [3] including the chamfered edges of the extraction apertures and the topology of the magnetic field, i.e. the superposition of the filter field and the field created by the electron deflection magnets.

TABLE 2. Reaction processes implemented into TrajAn.

\begin{tabular}{lcc}
\hline Reaction & Process & Ref. \\
\hline Electron stripping & $\mathrm{H}^{-}+\mathrm{e} \rightarrow \mathrm{H}+2 \mathrm{e}$ & {$[27]$} \\
Mutual neutralization with $\mathrm{H}^{+}$ & $\mathrm{H}^{-}+\mathrm{H}^{+} \rightarrow 2 \mathrm{H}$ & {$[28]$} \\
Mutual neutralization with $\mathrm{Cs}^{+}$ & $\mathrm{H}^{-}+\mathrm{Cs}^{+} \rightarrow \mathrm{H}+\mathrm{Cs}$ & {$[29]$} \\
Collisional detachment with $\mathrm{H}$ & $\mathrm{H}^{-}+\mathrm{H} \rightarrow \mathrm{H}+\mathrm{H}+\mathrm{e}$ & {$[27]$} \\
Collisional detachment with $\mathrm{H}_{2}$ & $\mathrm{H}^{-}+\mathrm{H}_{2} \rightarrow \mathrm{H}+\mathrm{H}_{2}+\mathrm{e}$ & {$[30]$} \\
Associative detachment & $\mathrm{H}^{-}+\mathrm{H} \rightarrow \mathrm{H}_{2}+\mathrm{e}$ & {$[27]$} \\
Charge exchange & $\mathrm{H}^{-}+\mathrm{H} \rightarrow \mathrm{H}+\mathrm{H}^{-}$ & {$[31]$} \\
\hline
\end{tabular}

Single test particles are started at the sheath edge. The motion of these test particles is divided into small time steps. For each time step the influence of the magnetic field on the particle trajectory is calculated. Then, a Monte Carlo module is invoked which checks whether a collision takes place or not. The seven collision processes included into TrajAn are listed in Table 2. For each time step also the influence of small angle Coulomb collisions on the particle trajectory is taken into account according to the method described in [32].

Several output routines exist in TrajAn which provide the user with information on the global extraction probability of the negative ions, the spatially resolved local 
extraction probability over the PG area and the current density distribution of negative hydrogen ions over the extraction aperture area.

\section{RESULTS}

Calculations with Protonflow3d have been performed for an ensemble of $10^{5}$ protons, the measured values for the density of the hydrogen atoms and molecules from [9] and the potential profile shown in figure 3.

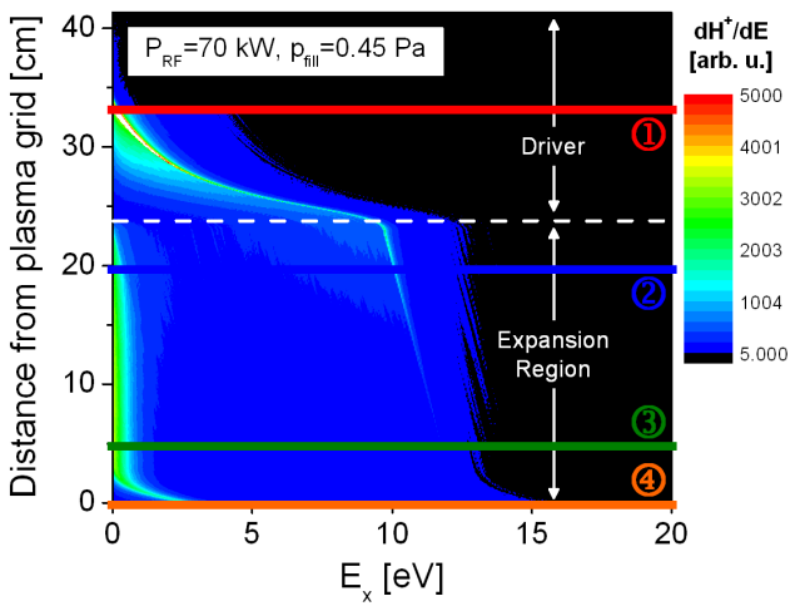

FIGURE 4. Phase space diagram for the transport of protons towards the PG during high power operation of the IPP prototype ion source.

The total mean free path for the six included collision processes is below a few centimeters. This means that the proton transport from the driver towards the PG is determined not solely by the electrostatic potential but also influenced considerably by collisions.

Figure 4 shows the distribution of the axial kinetic proton energy $E_{x}$ versus the distance from the PG for high power operation. The protons are started at $33 \mathrm{~cm}$ distance to the PG (at the marker (1) in figure 4) with an EDF representing a proton temperature of $0.8 \mathrm{eV}$. Towards the PG first a strong acceleration occurs caused by the potential difference between the driver and the expansion region (between markers (1) and (2)). The potential profile in the expansion region is more or less flat and almost no additional acceleration occurs. Due to the short collision mean free path, the proton EDF slowly thermalizes (between markers (2) and (3)). The most relevant collision processes are momentum transfer with $\mathrm{H}_{2}$ and $\mathrm{H}$ and rotational excitation of $\mathrm{H}_{2}$. At the boundary layer the EDF can be represented with good accuracy by a low temperature Maxwell distribution; the proton temperature is determined to be around $1.0 \mathrm{eV}$. A consecutive acceleration process is caused by the potential difference between plasma potential and bias potential at the PG surface when the protons approach the plasma sheath (between markers (3) and (4)).

For the subsequent investigations with Bacon on the physics of the plasma sheath and the surface generation of negative hydrogen ions a hydrogen atom temperature of $0.8 \mathrm{eV}$ is used. This value results from the measured Doppler broadening of the atomic $\mathrm{H}_{\alpha}$ emission line. For the protons the temperature $1.0 \mathrm{eV}$ calculated by 
Protonflow3d is applied. While for the atomic density again the result from [9] is used $\left(\mathrm{n}(\mathrm{H})=4.5 \cdot 10^{18} \mathrm{~m}^{-3}\right)$, the proton density is a direct result of the calculation process. Given as input is the plasma density to $\mathrm{n}_{\text {plasma }}=2 \cdot 10^{17} \mathrm{~m}^{-3}$, corresponding to the result of Langmuir probe measurements [10].

Figure 5 shows the profiles of the plasma potential and the densities of the charged particle densities calculated using Bacon. When comparing the potential profile with the one shown in Figure 3 it has to be kept in mind that the reference system of the PIC code uses the PG bias potential as zero-point. The physics of the plasma sheath is strongly influenced by the surface produced negative hydrogen ions. The high amount of negative hydrogen ions produced at the PG surface and their high mass cause a strong negative space charge close to the PG surface. As a result, the plasma potential is significantly lower compared to the results for identical plasma parameters but without surface production of negative ions. Additionally, a virtual cathode evolves at the PG and the emission of negative ions is negative space charge limited [24].

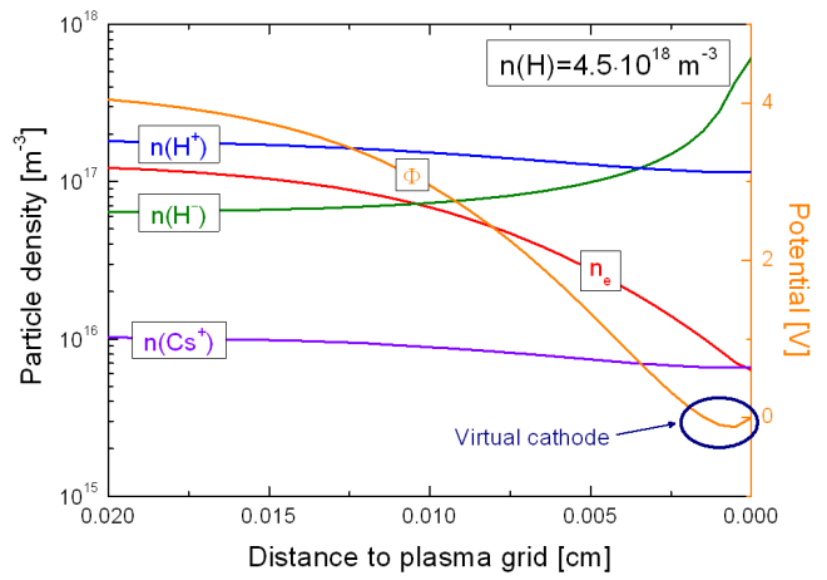

FIGURE 5. Profiles of the plasma potential (with the reference point, $\Phi=0 \mathrm{~V}$, at the PG surface) and the charged particle densities close to the PG.

The absolute negative hydrogen ion current density generated at the PG surface is $402 \mathrm{~A} / \mathrm{m}^{2}$. The virtual cathode is overcome by $80 \%$ of the surface generated negative ions, resulting in $323 \mathrm{~A} / \mathrm{m}^{2}$ emitted at the sheath edge. The relative relevance of the two different surface production channels is 3.6, i.e. the number of negative ions produced by conversion of atoms is 3.6 times higher compared to the conversion of protons. Bearing in mind that the calculations with Protonflow $3 d$ and Bacon have been performed without magnetic filter field an even higher ratio of the negative ion production channels is expected in the experiment.

Next, the correlation of the negative ion current density emitted from the sheath edge with the extracted ion current density is examined. For this purpose the extraction probability of negative hydrogen ions produced on the chamfered edge of a single aperture of the LAG system $\left(\varnothing_{\text {Aperture }}=8 \mathrm{~mm}\right.$. See also Figure $6 \mathrm{a}$, a sectional drawing of an extraction aperture) was calculated using TrajAn. The result for $7 \mathrm{mT}$ filter field strength and $1 \mathrm{eV}$ initial kinetic energy of the negative ions (i.e. the energy at the sheath edge) is shown in Figure 6b. The extraction probability shows a pronounced non-homogeneity over the area of the aperture edge, caused by the influence of the filter field. The average extraction probability is $34.6 \%$ [24]. 
Negative ion generation at the flat spots in between the chamfered edges is neglected due to the small area and the small extraction probability of these spots. The ratio of the negative ion production area (the chamfered edge) to the extraction area (the aperture) is equal to 1.25 . Thus, the negative ion current density emitted at the sheath edge has to be scaled by $34.6 \%$ and multiplied with 1.25 to determine the extracted current density. This means that of the $323 \mathrm{~A} / \mathrm{m}^{2}$ emitted at the sheath edge $140 \mathrm{~A} / \mathrm{m}^{2}$ are extracted, which agrees with experimental results from a reasonably well conditioned ion source.

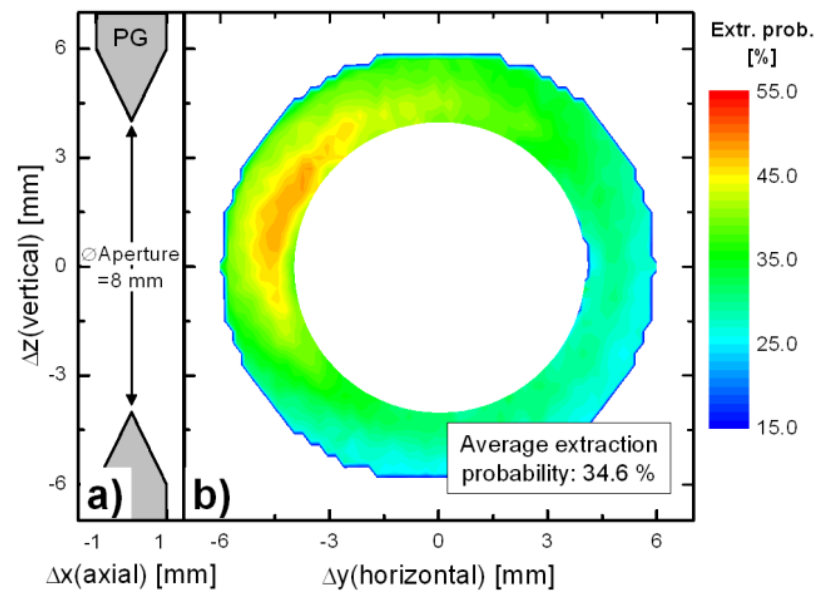

FIGURE 6. a) Sectional drawing of an extraction aperture. b) Extraction probability of negative ions produced at the chamfered edge of an aperture (for $\mathrm{B}=7 \mathrm{mT}$ und $\mathrm{E}_{\text {initial }}=1 \mathrm{eV}$ ).

The good agreement of this combined result of the three codes with the experiment emphasizes the reliability of the codes and their key results: Firstly, the protons approach the boundary layer with low energy due to collisional deceleration in the expansion region. Secondly, the high negative space charge close to the PG surface causes a virtual cathode. And thirdly, the conversion of atoms to negative ions is the much more relevant channel compared to the conversion of protons. This result explains the good beam homogeneity observed in [12].

\section{CONCLUSIONS}

Three different computer codes were applied in order to investigate the physics of the boundary layer in the IPP prototype negative ion source. One of the most important features of these codes is that they implement - as far as possible - the geometry, the plasma parameters and the topology of the electrostatic and magnetic fields of the experiment. The key aspect of the investigations is to compare the relevance of the two surface production channels for negative hydrogen ions: conversion of atoms and conversion of positive ions.

The first important result is that the protons accelerated by the potential difference between driver and expansion region approach the boundary layer with a low kinetic energy. This is caused by elastic and inelastic collisions with the background gas. Due to comparable velocities of hydrogen atoms and protons in the boundary layer and a high atomic density, the atomic flux onto the PG surface is significantly higher than the proton flux. The particle transport through the plasma sheath is influenced by a 
virtual cathode and the average extraction probability of negative ions produced on the chamfered edge of the extraction apertures is $34.6 \%$. The latter two effects affect the ions produced via the two surface production channels in the same way. As a consequence, the conversion of atoms is dominantly responsible for the production of the extracted negative hydrogen ions.

Thus, the final conclusion can be drawn that the neutral gas uniformity - during plasma operation affected by neutral depletion - represents a key parameter for the homogeneity of the negative ion beam.

\section{ACKNOWLEDGMENTS}

The work was supported by a grant from Fusion for Energy (F4E-2008-GRT-007) under the responsibility of Antonio Masiello. The opinions expressed herein are those of the authors only and do not represent the Fusion for Energy's official position.

\section{REFERENCES}

1. R. Hemsworth, A. Tanga and V. Antoni, Rev. Sci. Instrum. 79, 02C109 (2008).

2. R. Hemsworth, H. Decamps and J. Graceffa et al, Nucl. Fusion 49, 045006 (2009).

3. E. Speth, H. D. Falter and P. Franzen et al, Nucl. Fusion 46, S220 (2006).

4. U. Fantz, P. Franzen and W. Kraus et al, Rev. Sci. Instrum. 79, 02A511 (2008).

5. O. Fukumasa, IEEE Trans. Plasma Sci. 28, 1009 (2000).

6. V. Dudnikov, Rev. Sci. Instrum. 73, 992 (2002).

7. B. Rasser, J. N. M. van Wunnik and J. Los, Surf. Sci. 118, 697 (1982).

8. J. D. Isenberg, H. J. Kwon and M. Seidl, AIP Conf. Proc. 287, 38 (1994).

9. P. McNeely, D. Wünderlich and the NNBI-Team, Plasma Sources Sci. Technol. 20, 045005 (2011).

10.L. Schiesko et al, Plasma Phys. Control. Fusion 54, 105002, (2012).

11. U. Fantz, P. Franzen and W. Kraus et al, Plasma Phys. Control. Fusion 49, B563 (2007).

12.P. Franzen, U. Fantz and the NNBI-Team, AIP Conf. Proc. 1390, 310 (2011).

13. U. Fantz, P. Franzen, W. Kraus et al, Nucl. Fusion 49, 125007 (2009).

14.D. Wünderlich et al, accepted by Plasma Phys. Control. Fusion (2012).

15.P. McNeely, S. V. Dudin and S. Christ-Koch et al, Plasma Sources Sci. Technol. 18, 014011 (2009).

16. A. E. Glassgold, P. S. Krstic and D. R. Schultz, Astrophys. J. 621, 808 (2005).

17. T. Tabata and T. Shirai, At. Data Nucl. Data Tables 76, 1 (2000).

18. R. K. Janev, D. Reiter and U. Samm, Report JÜL-4105, Forschungszentrum Jülich (2003).

19. D. Wünderlich et al, The CR model Yacora for molecular hydrogen, in preparation (2012).

20.P. S. Krstic and D. R. Schultz, J. Phys. B: At. Mol. Opt. Phys. 32, 2415 (1999).

21. V. Hermann, H. Schmidt and F. Linder, J. Phys. B: At. Mol. Phys. 11, 493 (1978).

22. V. Vahedi and M. Surenda, Comp. Phys. Comm. 871, 179 (1995).

23. A. Tanga, M. Bandyopadhyay and P. McNeely, Appl. Phys. Lett. 84, 182 (2004).

24.D. Wünderlich, R. Gutser and U. Fantz, Plasma Sources Sci. Technol. 18, 045031 (2009).

25. C. K. Birdsall and A. B. Langdon, Plasma Physics via Computer Simulation, McGraw-Hill, New York (1985).

26.R. Gutser, D. Wünderlich, U. Fantz et al, Plasma Phys. Control. Fusion 51, 045005 (2009).

27.R. K. Janev, W. Langer and J. Evans et al, Elementary Processes in Hydrogen-Helium Plasmas, Springer, Berlin (1987).

28.M. J. J. Eeerden, M. C. M. van de Sanden, D. K. Otorbaev et al, Phys. Rev. A 51, 3362 (1994).

29.R. K. Janev and Z. Radulovic, Phys. Rev. A 17, 889 (1978).

30.C. F. Barnett, Report ORNL-6086, Oak Ridge National Laboratory (1990).

31.M. Huels, R. Champion R and Doverspike et al, Phys. Rev. A 41, 4809 (1990).

32.S. Ma, R. Sydora and J. Dawson, Comput. Phys. Commun. 77, 190 (1993). 